\title{
Study of charge Transport in Silicon Detectors: Non-Irradiated and Irradiated
}

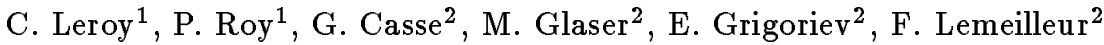 \\ 1 Université de Montréal, Montréal (Québec) H3C 3J7, Canada \\ ${ }^{2}$ CERN, Geneva, Switzerland
}

The electrical characteristics of silicon detectors (standard planar float zone and MESA detectors) as a function of the particle fluence can be extracted by the application of a model describing the transport of charge carriers generated in the detectors by ionizing particles. The current pulse response induced by $\alpha$ and $\beta$ particles in non-irradiated detectors and detectors irradiated up to fluences $\Phi \approx 3 \cdot 10^{14}$ particles $/ \mathrm{cm}^{2}$ is reproduced via this model: i) by adding a small $\mathrm{n}$-type region $15 \mu \mathrm{m}$ deep on the $p^{+}$side for the detectors at fluences beyond the $\mathrm{n}$ to p-type inversion and ii) for the MESA detectors, by considering one additional dead layer of $14 \mu \mathrm{m}$ (observed experimentally) on each side of the detector, and introducing a second (delayed) component to the current pulse response. For both types of detectors, the model gives mobilities decreasing linearily up to fluences of about $5 \cdot 10^{13}$ particles $/ \mathrm{cm}^{2}$ and converging, beyond, to saturation values of about $1050 \mathrm{~cm}^{2} / \mathrm{Vs}$ and $450 \mathrm{~cm}^{2} / \mathrm{Vs}$ for electrons and holes, respectively. At a fluence $\Phi \approx 10^{14}$ particles $/ \mathrm{cm}^{2}$ (corresponding to about ten years of operation at the CERN-LHC), charge collection deficits of about $14 \%$ for $\beta$ particles, $25 \%$ for $\alpha$ particles incident on the front and $35 \%$ for $\alpha$ particles incident on the back of the detector are found for both type of detectors.

\section{Introduction}

The signal response induced by the transport of the carriers of the charge generated by an incident particle in a silicon detector is governed by a set of basic equations. Solving this set of equations, a model is obtained from which the electrical characteristics of non-irradiated and irradiated silicon detectors can be extracted. The model is used to fit the experimental signal-current pulse responses (measured as a function of the collection time) induced by $\alpha$ and $\beta$ particles in $p^{+}-n-n^{+}$ silicon detectors. The electrical characteristics of a $p^{+}-n-n^{+}$detector extracted that way are the effective impurity or dopant concentration $\left(N_{e f f}\right)$, the electron $\left(\mu_{e}\right)$ and hole $\left(\mu_{h}\right)$ mobilities, and the charge carrier lifetimes $\left(\tau_{t e}, \tau_{t h}\right)$.

\section{The charge transport model}

The electrical characteristics are extracted from a system of five partial differential equations: the current continuity equations for electrons and holes, the Poisson equation (which determines the electric field and considers the plasma effect) and two equations relating the con- centration of trapped to the untrapped charges. As no analytical solution can be obtained, the equations are discretized using Gummel's decoupling scheme [1] to obtain a numerical solution [2]. The observed signal $(V(t))$ is a convolution of the current $(I(t)$ obtained from Ramo's theorem [3]) produced by all the individual charge carriers and the response from the system, which is simply an $\mathrm{RC}$ circuit. The response of the system is Gaussian with a characteristic time constant $\sigma=R_{a} C$, where $C$ is the capacitance of the detector and $R_{a}=50 \Omega$ the input impedance of the amplifier:

$$
\begin{aligned}
& I(t)=\frac{18 D_{a} t+r_{0}^{2}}{w r_{0}^{2}} \int_{0}^{w}\left(\mu_{e} n+\mu_{h} p\right) E d x \\
& V(t)=\frac{G R_{a}}{\sigma \sqrt{2 \pi}} \sum_{e, h} \int_{-\infty}^{\infty} I\left(t^{\prime}\right) e^{-\frac{\left(t-t^{\prime}\right)^{2}}{2 \sigma^{2}}} d t^{\prime}
\end{aligned}
$$

where $n$ and $p$ are the concentration of electrons and holes, $E$ the electric field, $D_{a}$ the ambipolar diffusion constant, $G=1000$ the gain of the amplifier, $r_{0}$ the initial radius of the column of deposited charge and $w$ the thickness of the detectors. The fact that the drift velocity of the charge carrier reaches a saturation value $v_{s}$ for 
Table 1

Characteristics of the detectors. The detectors were irradiated by step of fluence up to $9.92 \cdot 10^{13} \mathrm{n} / \mathrm{cm}^{2}$ for $\mathrm{M} 4,7.48 \cdot 10^{13} \mathrm{p} / \mathrm{cm}^{2}$ for M18, M25, M35, and up to $28.7 \cdot 10^{13} \mathrm{p} / \mathrm{cm}^{2}$ for P44, P86, P88, P135, P189, P300 and P304. Detectors M49, M50 and M53 were not irradiated

\begin{tabular}{|l|l|l|l|l|l|l|}
\hline Detector & Process & $\begin{array}{l}\text { Current } \\
\text { pulse source }\end{array}$ & $\begin{array}{l}\text { Thickness } \\
(\mu \mathrm{m})\end{array}$ & $\begin{array}{l}\mathrm{N}_{\text {eff }}(\Phi=0) \\
\left(10^{11} \mathrm{~cm}^{-3}\right)\end{array}$ & $\begin{array}{l}\text { Resistivity } \\
\mathrm{k} \Omega \cdot \mathrm{cm}\end{array}$ & $\begin{array}{l}\text { Maximum } \\
\text { fluence }\left(\mathrm{cm}^{-2}\right)\end{array}$ \\
\hline M4 & SPFZ & $\alpha$ & $\mathbf{3 1 7}$ & -3.4 & 12.2 & $9.92 \cdot 10^{13} \mathrm{n}$ \\
\hline M18 & SPFZ & $\alpha, \beta$ & 309 & -4.1 & 11. & $7.48 \cdot 10^{13} \mathrm{p}$ \\
\hline M25 & SPFZ & $\alpha, \beta$ & 308 & -2.1 & 23. & $7.48 \cdot 10^{13} \mathrm{p}$ \\
\hline M35 & SPFZ & $\alpha$ & 508 & -1.7 & 24. & $7.48 \cdot 10^{13} \mathrm{p}$ \\
\hline M49 & SPFZ & $\beta$ & 301 & -4.7 & 8.9 & - \\
\hline M50 & SPFZ & $\beta$ & 471 & -1.8 & 22.8 & - \\
\hline M53 & SPFZ & $\beta$ & 223 & -5.4 & 7.7 & - \\
\hline P44 & MESA & $\alpha, \beta$ & 306 & -18 & 2. & $28.7 \cdot 10^{13} \mathrm{p}$ \\
\hline P86 & SPFZ & $\alpha, \beta$ & 290 & -21 & 2.5 & $28.7 \cdot 10^{13} \mathrm{p}$ \\
\hline P88 & SPFZ & $\alpha, \beta$ & 290 & -19 & 2.5 & $28.7 \cdot 10^{13} \mathrm{p}$ \\
\hline P135 & MESA & $\alpha, \beta$ & $\mathbf{3 0 8}$ & -17 & 2. & $28.7 \cdot 10^{13} \mathrm{p}$ \\
\hline P189 & SPFZ & $\alpha, \beta$ & 294 & -21 & 2.5 & $28.7 \cdot 10^{13} \mathrm{p}$ \\
\hline P300 & MESA & $\alpha, \beta$ & $\mathbf{3 0 3}$ & -6 & 6. & $28.7 \cdot 10^{13} \mathrm{p}$ \\
\hline P304 & SPFZ & $\alpha, \beta$ & $\mathbf{3 2 0}$ & -6 & 6. & $28.7 \cdot 10^{13} \mathrm{p}$ \\
\hline
\end{tabular}

electric field values around $10^{4} \mathrm{~V} / \mathrm{cm}$ has been taken into account [2], as well as the dependence on the temperature and dopant concentrations via the empirical equation:

$\mu\left(T, N_{e f f}\right)=\mu_{\min }+\frac{\mu_{0}\left(\frac{T}{300}\right)^{\nu}-\mu_{\min }}{1+\left(\frac{T}{300}\right)^{\xi}\left(\frac{N_{e f f}}{N_{\text {ref }}}\right)^{\alpha}}$

where the values used for the electrons (holes) are: $\mu_{\min }=55.24(49.7) \mathrm{cm}^{2} / \mathrm{Vs}, N_{\text {ref }}=1.072 \cdot 10^{17}$ $\left(1.606 \cdot 10^{17}\right)$ dopants $/ \mathrm{cm}^{3}, \nu=-2.3(-2.2), \xi=$ $-3.8(-3.7), \alpha=0.73(0.70), T$ is the temperature in Kelvin and $\mu_{0}$ is the mobility at $T=300 \mathrm{~K}$.

The quantities of interest are extracted by using the code MINUIT [4] to minimize the $\chi^{2}$ obtained from fitting the numerical solutions found for $V(t)$ to the measured current pulse response induced by $\alpha$ and $\beta$ particles.

The current pulses were induced by electrons from a ${ }^{106} \mathrm{Ru}$ source with an energy $>2 \mathrm{MeV}$, selected by an external trigger, and $\alpha$ particles from a ${ }^{241} \mathrm{Am}$ source with an energy of $5.49 \mathrm{MeV}$. The current pulses are detected by a fast current amplifier and recorded by a digital oscilloscope used in averaging mode, to improve the signalto-noise ratio. The detectors used in the present work are listed in Table 1 with their thickness and resistivity before irradiation.

\section{Non-irradiated detectors}

Figure 1 gives a representation of a standard planar float zone (SPFZ) detector. The $\mathrm{p}^{+}$and $\mathrm{n}^{+}$regions are neglected. Fits of the charge transport model to the current pulses induced by $\alpha$ and $\beta$-particles in non-irradiated SPFZ detectors are shown in Fig. 2. The model reproduces well the shape of the measured current pulses.

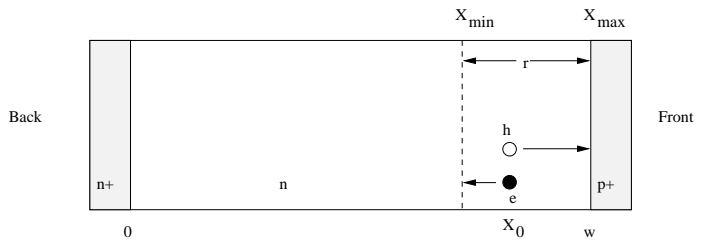

Figure 1. Schematic representation of a SPFZ detector.

The schematic representation of a MESA detector is shown in Fig. 3. We start with a MESA detector of thickness $w$, of which a thickness $x_{\text {dead }}$ is considered dead on each side, thus the active thickness of the detector is $w^{\prime}=w-2 \cdot x_{\text {dead }}$. 

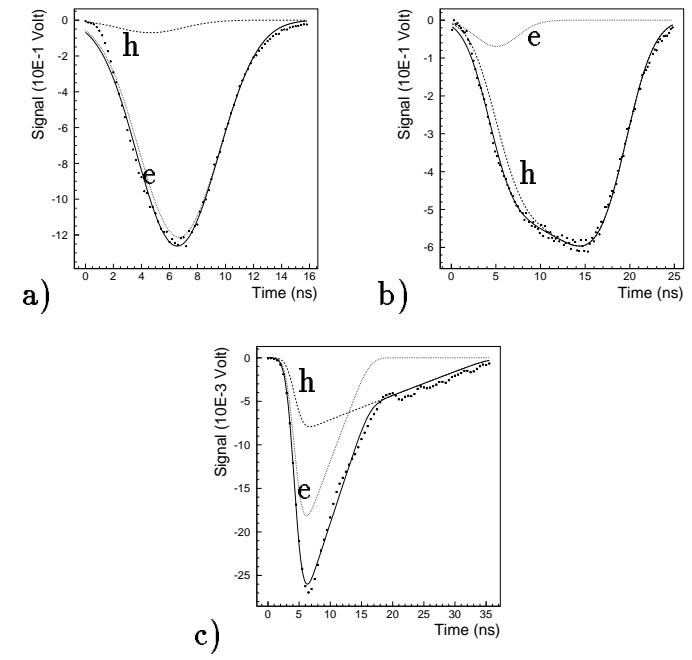

Figure 2. Fits (full line) of the charge transport model to the current pulse response induced at $\Phi=0$ by $\alpha$ particles incident on the front side (a), on the back side (b) of detector M25 and by relativistic electrons on detector M50 (c); A bias voltage $V_{b}=160 \mathrm{~V}$ is applied in all cases. Electrons (e) and holes (h) contributions are shown.

Indeed, studies of MESA detectors have shown that a layer of $\approx 14 \mu \mathrm{m}$ on each side of the detectors acts as a dead layer [5]. The initial distribution of charge carriers at time $t=0$ over the total thickness $w$ is the same as for a SPFZ detector, even though only the active region $x_{\text {dead }}<x<w-x_{\text {dead }}$ will generate the signal in a MESA detector.

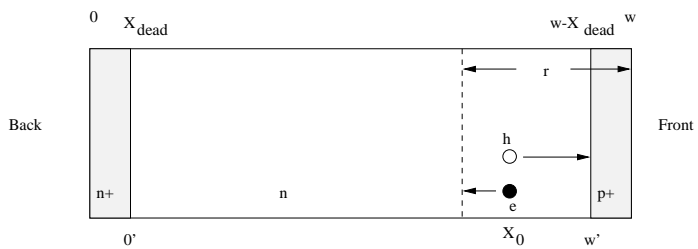

Figure 3. Schematic representation of a MESA detector.

Figure 4a shows that for MESA detectors, the inclusion of a dead layer (observed experimentaly) $14 \mu \mathrm{m}$ deep on each side of the detector alone is not enough for the model to reproduce the current-pulse. The problem is solved by considering that a fraction $(f)$ of the electron-hole pairs created in the dead zones becomes active only at a later time $T_{\text {late }}$ which gives a second component (line 2 in Fig. 4b). For $\alpha$ particles incident on the front (back) side of the detector, some electrons (holes) will be released near the front (back), while for $\beta$ particles a combination of both cases happens. A very good description of the measured current-pulse of a MESA detector is obtained by adding the two components (line 3 in Fig. 4b).
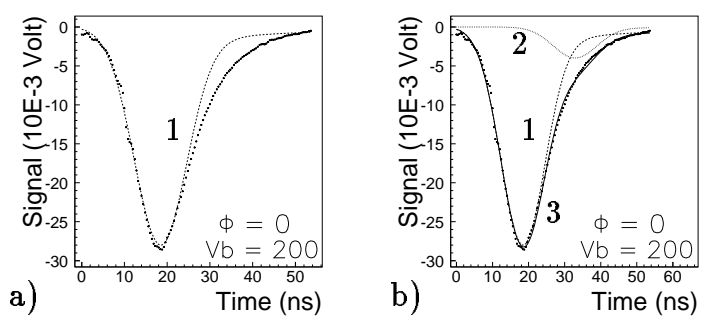

Figure 4. Current pulse response induced by $\alpha$ particles incident on the back of a MESA detector, before (line 1 ) and after (line 3 ) the addition of a second (delayed) component (line 2) at $\Phi=0$ and applied voltage $V_{b}=200$ Volts.

Table 2

Mobilities at $\Phi=0$ of the SPFZ and MESA detectors as obtained from the model

\begin{tabular}{|l|l|l|l|}
\hline Detector & Process & $\begin{array}{l}\mu_{h} \\
\left(\mathrm{~cm}^{2} / \mathrm{Vs}\right)\end{array}$ & $\begin{array}{l}\mu_{e} \\
\left(\mathrm{~cm}^{2} / \mathrm{Vs}\right)\end{array}$ \\
\hline M4 & SPFZ & $504 \pm 2$ & $1278 \pm 15$ \\
\hline M18 & SPFZ & $474 \pm 2$ & $1236 \pm 15$ \\
\hline M25 & SPFZ & $476 \pm 2$ & $1308 \pm 28$ \\
\hline M35 & SPFZ & $472 \pm 3$ & $1272 \pm 5$ \\
\hline M49 & SPFZ & $546 \pm 11$ & $1266 \pm 24$ \\
\hline M50 & SPFZ & $529 \pm 13$ & $1272 \pm 20$ \\
\hline M53 & SPFZ & $478 \pm 12$ & $1350 \pm 20$ \\
\hline P44 & MESA & $455 \pm 15$ & $1422 \pm 24$ \\
\hline P88 & SPFZ & $459 \pm 4$ & $1222 \pm 20$ \\
\hline P135 & MESA & $472 \pm 9$ & $1310 \pm 23$ \\
\hline P189 & SPFZ & $480 \pm 20$ & $1340 \pm 27$ \\
\hline P300 & MESA & $469 \pm 12$ & $1298 \pm 18$ \\
\hline P304 & SPFZ & $495 \pm 3$ & $1124 \pm 22$ \\
\hline
\end{tabular}

Table 2 shows the initial mobilities (at $\Phi=0$ ) of the SPFZ and MESA detectors obtained from the model. The average mobilities achieved for electrons and holes are: $\mu_{e}=1284 \pm 21 \mathrm{~cm}^{2} / \mathrm{Vs}$ and $\mu_{h}=482 \pm 10 \mathrm{~cm}^{2} / \mathrm{Vs}$, respectively. 

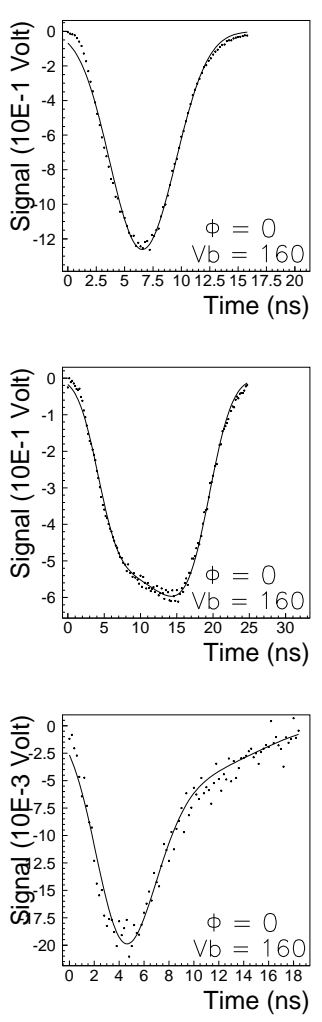

alpha front
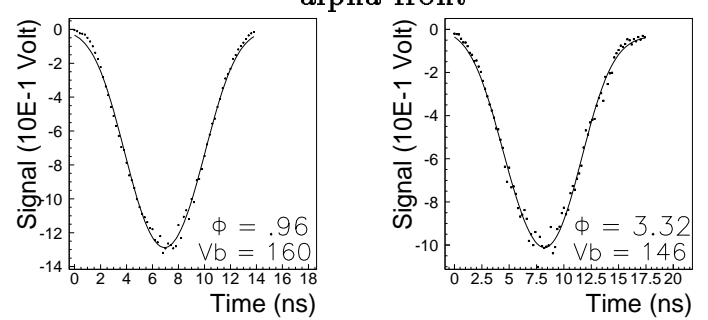

alpha back
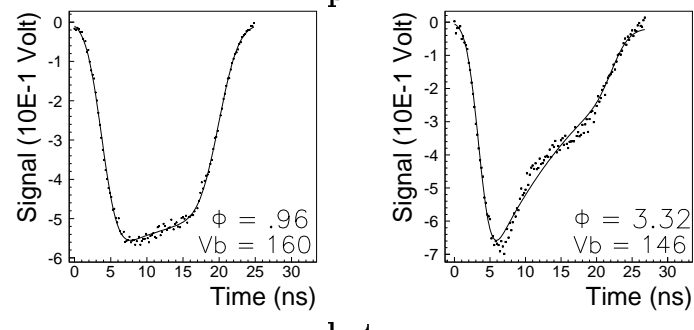

beta

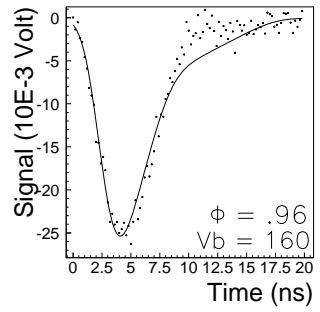

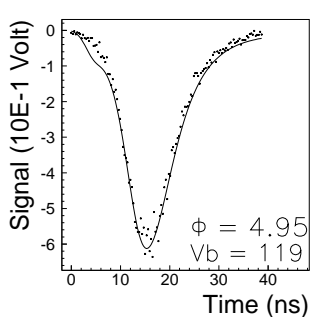
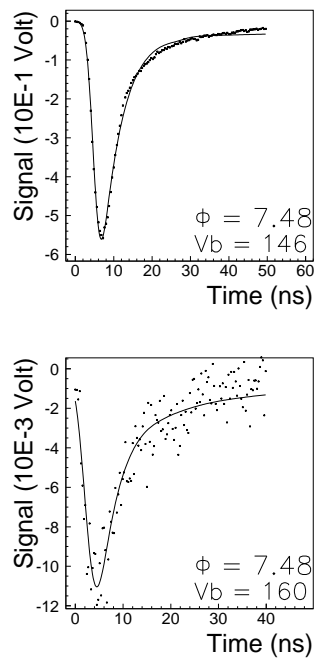

Figure 5. Fits (full line) of the current pulse response induced by $\alpha$ particles incident on the front side (first row), back side (second row) or from particles $\beta$ (third row) on the detector M25 for successive levels of fluence $\Phi$ from 0 up to $7.48 \cdot 10^{13} \mathrm{p} / \mathrm{cm}^{2}$ ( $\Phi$ in $10^{13} \mathrm{p} / \mathrm{cm}^{2}, V_{b}$ is the applied voltage in volts).

\section{Irradiated detectors}

The irradiations of the detectors were performed either at the CERN-PSAIF with $\approx 1 \mathrm{MeV}$ neutrons, up to a fluence of $9.92 \cdot 10^{13} \mathrm{n} / \mathrm{cm}^{2}$ or at the CERN-PS with $24 \mathrm{GeV} / \mathrm{c}$ protons, up to a fluence of $2.87 \cdot 10^{14} \mathrm{p} / \mathrm{cm}^{2}$ (column 7 in Table 1 ).

As described in [2], in order to fit the data and to account for the evolution of the electrical characteristic of the detectors with fluence beyond the $\mathrm{n}$ to $\mathrm{p}$-type inversion, the electric field is modified after inversion by introducing a $15 \mu \mathrm{m}$ n-type region near the $p^{+}$contact. This concept of double junction can be also found in $[6]$ and in other references contemporary with the present work $[7,8]$.

The evolution of the current pulse response of a SPFZ detector (M25) with fluence, as described by the model, is shown in Fig. 5 .
The evolution as a function of fluence of a MESA detector ( $\mathrm{P} 44$ ) current pulse response obtained by considering one dead layer $14 \mu \mathrm{m}$ deep on each side and introducing a second (delayed) component to the current pulse is shown in Fig. 6 .

As it can be seen from Fig.7, the mobilities obtained for SPFZ and MESA detectors are found to be in very good agreement. For both type of detectors, the mobility tends, after an initial decrease, towards the saturation values $\mu_{\text {sat } e} \approx$ $1050 \mathrm{~cm}^{2} / \mathrm{Vs}$ and $\mu_{s a t, h} \approx 450 \mathrm{~cm}^{2} / \mathrm{Vs}$ for the electrons and holes for $\Phi>5 \cdot 10^{13}$ particles $/ \mathrm{cm}^{2}$, respectively. This figure also shows that the mobility values obtained using either $\alpha$ or $\beta$ particles data are in agreement, which provides a consistency check of the model.

The results of the charge transport model fits to the experimental data permit the extraction of 

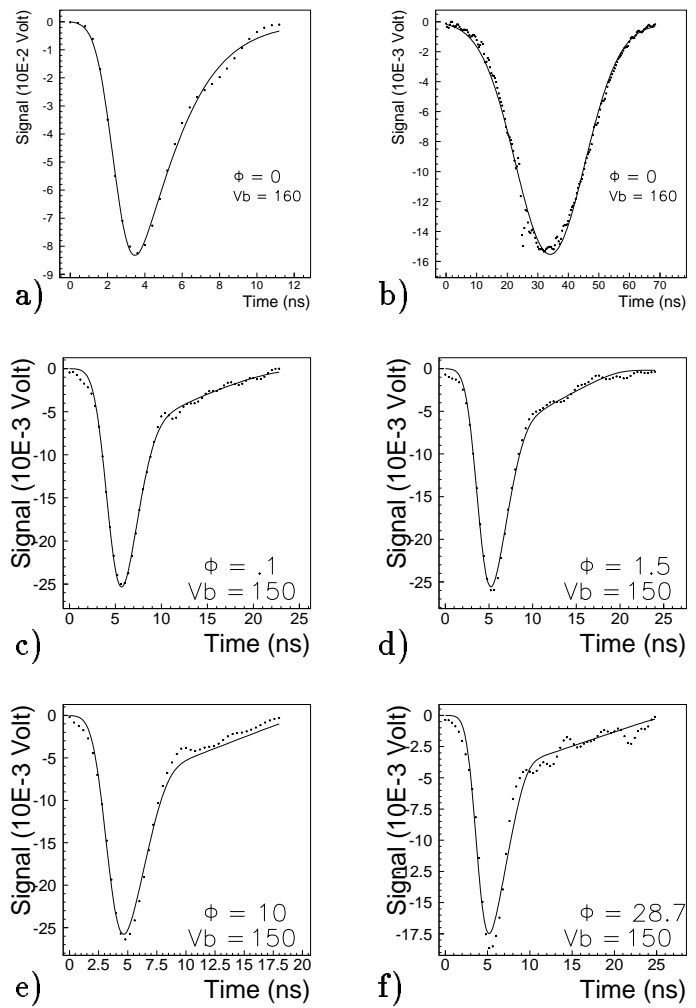

Figure 6. Fits (full line) of the current pulse response induced by $\alpha$ particles incident on the front side (a), on the back side (b) and for $\beta$ particles (c-f) of MESA detector P44 for successive levels of fluence $\Phi$ from 0 up to $2.87 \cdot 10^{14} \mathrm{p} / \mathrm{cm}^{2}$ ( $\Phi$ is in units of $10^{13} \mathrm{p} / \mathrm{cm}^{2}, V_{b}$ is the applied voltage in volts).

the value of $N_{\text {eff }}$ as a function of the fluence:

$N_{e f f}=-N_{d} \exp (-c \Phi)+N_{a}+b \Phi$,

where $N_{d}$ and $N_{a}$ are the concentration of donnors and acceptors at $\Phi=0$, respectively; $b$ and $c$ are the acceptor creation and donnor removal parameters, respectively. By using Eq. (4) to describe the evolution of $N_{\text {eff }}$ with fluence, one obtains the results shown in Fig. 8 for the detectors P44, P88, P189 and P304.
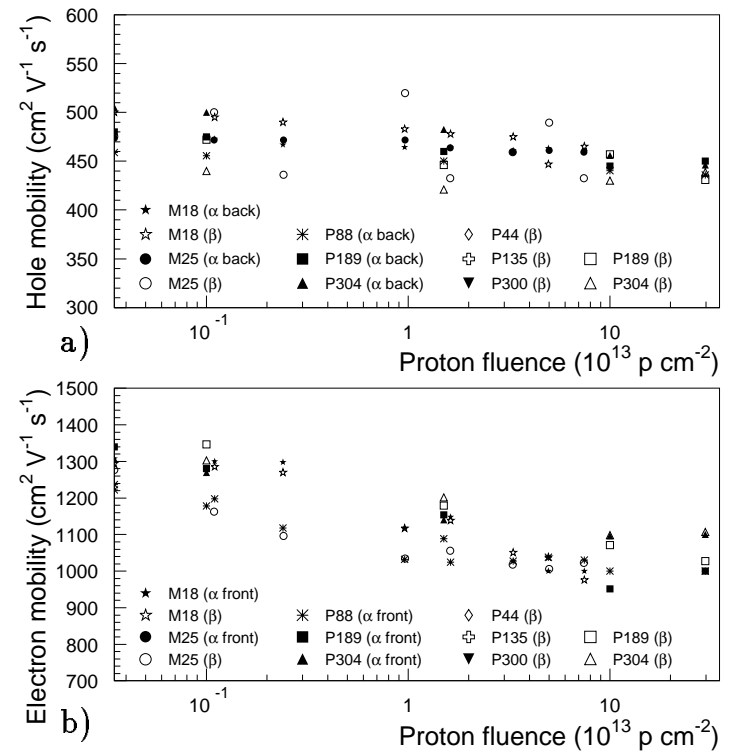

Figure 7. Evolution of the hole (a) and electron (b) mobility of SPFZ and MESA detectors as a function of fluence, as extracted from the model using $\alpha$ (front and back) and $\beta$ particles data.

\section{Charge collection efficiency}

A comparison between the results obtained using the trapping lifetime extracted at a certain fluence, with those obtained if no trapping had occured, allows the calculation of the charge collection efficiency (CCE). As it can be seen from Fig $9 \mathrm{a}, 9 \mathrm{~b}$, for neutron (proton) irradiated SPFZ detectors, a charge collection deficit around $12 \%$ $(25 \%)$ is calculated for $\alpha$ particles incident on the front side and about $18 \%$ (35\%) for $\alpha$ particles incident on the back side of the detector, for a fluence of $\approx 10^{14}$ particles $/ \mathrm{cm}^{2}$. Direct measurements [9] of CCE using $\alpha$ particles from a Th C' source with an energy of $8.78 \mathrm{MeV}$ on detectors irradiated up to a fluence of $\approx 10^{14}$ protons $/ \mathrm{cm}^{2}$ show a smaller deficit $(\approx 5 \%$ on the front side and $\approx 10 \%$ on the back side). Those discrepancies can be explained. First, an $\alpha$ particle of 5 (8.78) $\mathrm{MeV}$ has a range of $\approx 25(57)$ microns in silicon, most of the energy being deposited toward the end of the path. We can assume that most electron-hole pairs are created around 20 

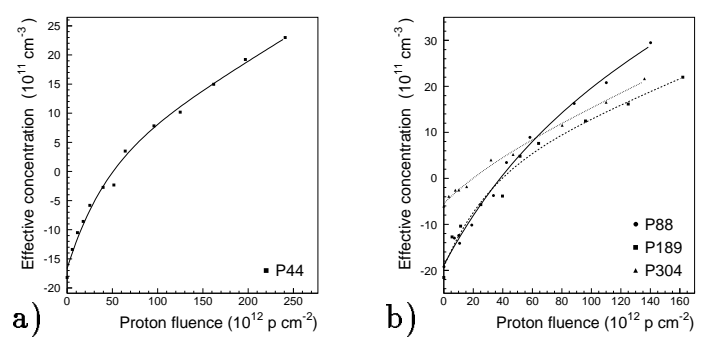

Figure 8. Evolution of the effective concentration of dopants as a function of fluence for the MESA detector P44 and the SPFZ detectors P88, P189 and P304.

(50) microns from the surface. Thus, for a typical detector of 300 microns, the charge carriers generated by $\alpha$ particles from an ${ }^{241} \mathrm{Am}$ source will experience more trapping as they spend $\approx 15 \%$ more time in the detector than those generated by $\alpha$ particles from a Th C' source. Secondly, the setup used with the Th C' source in ref. [9] had a shaping time ( $1 \mu \mathrm{s})$ larger than the shaping time (100 ns) used with the present ${ }^{241} \mathrm{Am}$ source setup. A large shaping time means that the trapped charges are more likely to untrap and thus reduce the observed charge collection deficit.

For $\beta$ particles on SPFZ detectors, a collection deficit of about $15 \%$ is calculated (Fig. 9c) for a fluence of $\approx 10^{14}$ particles $/ \mathrm{cm}^{2}$. For MESA detectors, a collection deficit of about $13 \%$ and 17 $\%$ are calculated (Fig. $9 \mathrm{~d}$ ) for fluences of $\approx 1$ and $3 \cdot 10^{14}$ particles $/ \mathrm{cm}^{2}$, respectively. Those results are in agreement with the $12 \%$ deficit obtained from direct charge collection efficiency measurements made with $\beta$ particles (shaping time of 100 ns) $[10]$ for SPFZ detectors irradiated at fluences $\approx 10^{14}$ particles $/ \mathrm{cm}^{2}$.

\section{Conclusions}

The model describing the transport of the carriers of charge generated in silicon detectors by ionizing particles allows one to reproduce the current pulse response of non-irradiated and irradiated SPFZ and MESA detectors induced by $\alpha$ and $\beta$ particles up to fluences around $n$ to $\mathrm{p}$-type inversion using a simple $p^{+}-n-n^{+}$detector. Beyond inversion a small $\mathrm{n}$-type region $15 \mu \mathrm{m}$ deep is introduced on the $p^{+}$side of the detector. The
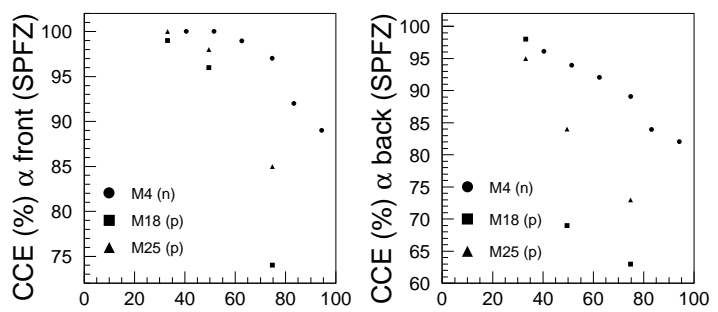

a) $\Phi\left(10^{12}\right.$ particles $\left.\mathrm{cm}^{-2}\right)$

b) $\Phi\left(10^{12}\right.$ particles $\left.\mathrm{cm}^{-2}\right)$

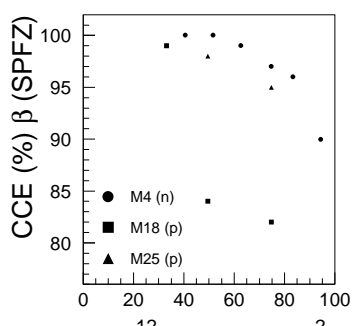

c) $\Phi\left(10^{12}\right.$ particles $\left.\mathrm{cm}^{-2}\right)$

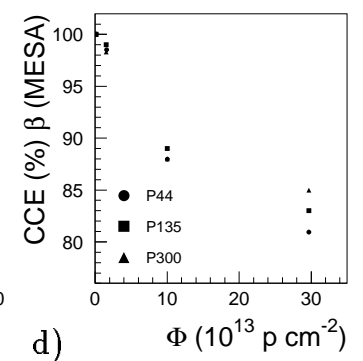

Figure 9. Charge collection efficiency as a function of fluence using: a) $\alpha$ particles incident on the front (SPFZ), b) $\alpha$ particles incident on the back (SPFZ), c) $\beta$ particles (SPFZ) and d) $\beta$ particles (MESA).

introduction of this region modifies the electric field after inversion and permits the charge carriers transport model to reproduce the experimental data up to fluences of $3 \cdot 10^{14}$ particles $/ \mathrm{cm}^{2}$. For MESA detector, a dead layer of $14 \mu \mathrm{m}$ (observed experimentaly) on each side of the detector is introduced, and a second (delayed) component is added to the current pulse response.

This model gives mobilities for SPFZ and MESA detectors in good agreement. The mobilities are found to be decreasing linearily up to fluences of around $5 \cdot 10^{13}$ particles $/ \mathrm{cm}^{2}$ and beyond, converging to saturation values of about $1050 \mathrm{~cm}^{2} / \mathrm{Vs}$ and $450 \mathrm{~cm}^{2} / \mathrm{Vs}$ for electrons and holes, respectively.

At a fluence $\Phi \approx 10^{14}$ particles $/ \mathrm{cm}^{2}$, the charge carrier lifetime degradation due to trapping with increased fluence is responsible for a charge collection deficit of about $14 \%$ for $\beta$ particles, $25 \%$ for $\alpha$ particles incident on the front side and $35 \%$ for $\alpha$ particles incident on the back side of SPFZ and MESA detectors, which is in agreement with direct measurements. 


\section{REFERENCES}

1. H. K. Gummel, A self-consistent iterative scheme for one-dimensional steady state transistor calculations, IEEE Trans. Electron. Devices ED-11 (1964) 455.

2. C. Leroy et al. Charge Transport in NonIrradiated and Irradiated Silicon Detectors, talk given at the International Conference on Radiation Effects on Semiconductor Materials, Detectors and Devices, Florence, Italy, 4 - 6 March 1998, to be published in Nucl. Instr. and Meth. A.

3. S. Ramo, Currents induced by electron motion, Proc. IRE vol. 27(9) (1939) 584.

4. Application Software Group, MINUIT Function Minimization and Error Analysis Reference Manual, Version 92.1, i, (1992).

5. G. Casse et al., Impact of mesa and planar processes on radiation hardness of Si detectors, CERN-ECP/97-09, January 1998.

6. Z. Li and H.W. Kraner, Fast neutron radiation damage effects on high resistivity silicon junction detectors, Journ. of Elec. Mat. vol. 21(7) (1992) 701

7. L.J. Beattie et al., The electric field in irradiated silicon detectors, ATLAS note INDETNO-194, 9 Dec. 1997.

8. D. Menichelli et al., Modelling of observed double junction effect, International Conference on Radiation Effects on Semiconductor Materials, Detectors and Devices, Florence, Italy, March 1998, to be published in Nucl. Instr. and Meth. A.

9. S. Pospísil, Scanning of silicon detector using alpha particles and low energy protons, talk given at GaAs'98, Pruhonice, 22-26 june 1998 , to be published in Nucl. Instr. and Meth. A (1998).

10. C. Leroy et al., Study of electrical properties and charge collection of silicon detectors under neutron, proton and gamma irradiations, Proc. IVth Int. Conf. on Calorimetry in High Energy Physics, World Scientific, eds. A. Menzione and A. Scribano, Singapore (1994) 627. 